\title{
A NONLOCAL PHASE-FIELD SYSTEM WITH INERTIAL TERM
}

\author{
BY \\ MAURIZIO GRASSELLI (Dipartimento di Matematica, Politecnico di Milano, Via E. Bonardi, 9, \\ I-20133 Milano, Italy), \\ HANA PETZELTOVÁ (Mathematical Institute AS CR, Žitná, 25, CZ-115 67, Praha 1, Czech \\ Republic), \\ AND
}

GIULIO SCHIMPERNA (Dipartimento di Matematica, Università di Pavia, Via Ferrata, 1, I-27100 Pavia, Italy)

\begin{abstract}
We study a phase-field system where the energy balance equation has the standard (parabolic) form, while the kinetic equation ruling the evolution of the order parameter $\chi$ is a nonlocal and nonlinear second-order ODE. The main features of the latter equation are a space convolution term which models long-range interactions of particles and a singular configuration potential that forces $\chi$ to take values in $(-1,1)$. We first prove the global existence and uniqueness of a regular solution to a suitable initial and boundary value problem associated with the system. Then, we investigate its long time behavior from the point of view of $\omega$-limits. In particular, using a nonsmooth version of the Lojasiewicz-Simon inequality, we show that the $\omega$-limit of any trajectory contains one and only one stationary solution, provided that the configuration potential in the kinetic equation is convex and analytic.
\end{abstract}

1. Introduction. Let $\Omega$ be a bounded and connected domain in $\mathbb{R}^{d}, 1 \leq d \leq 3$, with a smooth boundary $\Gamma:=\partial \Omega$. We consider the following coupled system in $\Omega \times(0,+\infty)$ :

$$
\begin{aligned}
& \vartheta_{t}+\chi_{t}-\Delta \vartheta=f, \\
& \varepsilon \chi_{t t}+\chi_{t}+W^{\prime}(\chi)=\mathcal{J}[\chi]+\vartheta,
\end{aligned}
$$

where $\varepsilon>0$ is a given constant.

Received May 8, 2006.

2000 Mathematics Subject Classification. Primary 34D05, 35B40, 35Q99, 80A22.

This work was partially supported by the Italian PRIN Research Project Problemi a frontiera libera, transizioni di fase e modelli di isteresi.

The work of the second author was supported by the Academy of Sciences of the Czech Republic, Institutional Research Plan no. AV0Z10190503 and by Grant IAA1001190606 of GA AV ČR.

The work of the last author was partially supported by the HYKE Research Training Network.

E-mail address: maurizio.grasselli@polimi.it

E-mail address: petzelt@math.cas.cz

E-mail address: giusch04@unipv.it 
This system describes the evolution of a two-phase material located in the container $\Omega$. It is endowed with given initial conditions and with homogeneous Dirichlet boundary condition for $\vartheta$, even though other boundary conditions might be considered. The unknowns $\vartheta$ and $\chi$ represent the (relative) temperature and the order parameter (or phase-field), respectively. The term $f$ in (1.1) is a (volumic) heat source, possibly depending on time, and $W$ is the (density of) potential energy associated with the phase configuration. More precisely, the values $\chi= \pm 1$ represent the pure states and $\chi \in(-1,1)$ stands for the local presence of a mushy region. The values outside $[-1,1]$ have no physical meaning and must be somehow penalized. To exclude them, we actually assume $W$ to be a (Lipschitz perturbation of) a convex and lower semicontinuous function which is identically $+\infty$ outside $(-1,1)$. We refer to this choice as that of a singular potential in the sequel (see also Remark 2.3 below). The operator $\mathcal{J}$ on the right hand side of (1.2) is defined by the relation

$$
\mathcal{J}[v](x)=\int_{\Omega} k(x-y) v(y) \mathrm{d} y,
$$

where $k: \mathbb{R}^{d} \rightarrow \mathbb{R}$ is an even and sufficiently smooth convolution kernel. The presence of $k$ is aimed to take into account long-range interactions between molecules or particles in the material. Although these interactions are physically justified and observable (e.g., in phase transition phenomena), in the mathematical literature, for many years, the operator $\mathcal{J}[\chi]$ was replaced by a diffusion term like $\nu \Delta \chi$, where $\nu$ is a positive parameter related to the thickness of the interface between the components [7] (cf. also [6]). This almost exclusive attention towards the "diffusive" models was probably motivated more by the purpose of simplifying the mathematical analysis rather than by physical reasons. Indeed, the relevance of nonlocal interaction phenomena in phase separation models was already clear in the pioneering paper [8]. Nevertheless, more recently, phase-field and phase-separation models containing nonlocal terms like (1.3) have known a growing interest and have been analyzed in many contributions (see, for instance, $[3,4,5,12,13$, $14,16,17,26,27,28,32,33])$. In these papers, the equation governing the evolution of the phase variable is essentially (1.2) (or its conserved version) with $\varepsilon=0$. In this case, we recall that, when $\mathcal{J}[\chi]$ is replaced by $\nu \Delta \chi$, we are dealing with a phase field model of parabolic type (see, e.g., [22]), where the phase relation is obtained as a gradient flow problem of the form

$$
\chi_{t}=-\delta_{\chi} E(\vartheta, \chi)
$$

$\delta_{\chi}$ being the variational derivative with respect to $\chi$ of the free energy $E$, given (in the diffusive case) by

$$
E(\vartheta, \chi)=\int_{\Omega}\left(\frac{\nu}{2}|\nabla \chi(x)|^{2}+W(\chi(x))-\vartheta(x) \chi(x)\right) \mathrm{d} x .
$$

In this regard, the term on the right hand side of (1.4) can be interpreted as a sort of generalized force driving the evolution of $\chi$, that is, the phase transformation. However, for certain materials, it can be experimentally observed that their responses to this force are not completely instantaneous, but characterized by some "delay" or "memory" effect (see, for instance, [15] and references therein). The simplest way to account for this effect 
is to add an inertial term, that is, to replace (1.4) by

$$
\varepsilon \chi_{t t}+\chi_{t}=-\delta_{\chi} E(\vartheta, \chi)
$$

for, e.g., $\varepsilon \in(0,1]$. In the case of the energy given by (1.5), equation (1.6) gives rise to the so-called parabolic-hyperbolic phase-field model which has been recently analyzed in a number of papers (see [19, 20, 21, 23, 34]). Instead, if the energy (1.5) is replaced by the "nonlocal" analog

$$
E_{\mathrm{n} 1}(\vartheta, \chi)=\int_{\Omega}\left(\int_{\Omega}-k(x-y) \frac{\chi(x) \chi(y)}{2} \mathrm{~d} y+W(\chi(x))-\vartheta(x) \chi(x)\right) \mathrm{d} x,
$$

then the relaxed gradient flow (1.6) entails just (1.2). To realize that the above expression models long range interactions, it is helpful to rewrite the first term on the right hand side as

$$
\int_{\Omega} k(x-y) \frac{|\chi(x)-\chi(y)|^{2}}{4} \mathrm{~d} y-\frac{\kappa(x) \chi^{2}(x)}{2},
$$

where $k$ is smooth enough in order to ensure that $\kappa(x):=\int_{\Omega} k(x-y) \mathrm{d} y$ is bounded and continuous. Note that, in one dimension, the nonlocal term in (1.8) can be approximated by the derivative of $\chi$ at $x$, provided that $k$ is peaked around 0 , recovering an approximated free energy similar to (1.5).

As we mentioned above, in the diffusive case, parabolic-hyperbolic phase-field systems have been extensively analyzed both from the point of view of well-posedness and of the long time behavior of solutions. In particular, in [20, 21, 23, 34] the singular potential is always replaced by a smooth approximation like $W(r)=\left(r^{2}-1\right)^{2}, r \in \mathbb{R}$. In [19], a singular potential is considered, provided that $\varepsilon$ is small enough. This restriction is not necessary here. In fact, our treatment of the singular potential is simpler since the Laplace operator is replaced by the nonlocal operator $\mathcal{J}$, so that the equation for $\chi$ has to be analyzed by ODE techniques.

Our first result on (1.1)-(1.2) concerns the well-posedness of the system in a suitable regularity setting. Since we want to treat (1.2) essentially as an ODE, it is convenient to work with bounded solutions. This is somehow in contrast with the diffusive case, where one usually works with solutions of bounded energy (i.e., such that $\left.W(\chi) \in L^{\infty}\left(0, \infty ; L^{1}(\Omega)\right)\right)$. We note that asking that both $\chi$ and $W(\chi)$ stay bounded in the $L^{\infty}$ norm corresponds to saying that $\chi$ satisfies almost everywhere a separation property of the form $-1+\delta \leq \chi \leq 1-\delta$ for some $\delta>0$, which actually permits us to get rid of the mathematical difficulties connected with the singular character of $W$ at \pm 1 (see also [22] and references therein). Of course, to implement this approach, we need to deal with a sufficiently smooth $\vartheta$ in order to have $\vartheta(t) \in L^{\infty}(\Omega)$ for almost any $t \geq 0$. It is also worth pointing out that, as observed in [12] (which deals with system (1.1)-(1.2) with $\varepsilon=0$ ), the precise expression (1.3) for the operator $\mathcal{J}$ plays no role in the mathematical analysis. What seems necessary is that $\mathcal{J}$ be a linear operator defined on $L^{2}(\Omega)$ satisfying some compactness properties (see (J1)-(J2) below). If $\mathcal{J}$ is a convolution operator as in (1.3), this just means that the kernel $k$ has to fulfill some additional growth and smoothness properties, depending on the space dimension, so that $\mathcal{J}$ can have a regularizing effect. In particular, kernels which are too singular in a neighborhood of 0 are not allowed (see, e.g., [14, Rem. 2.2] for some meaningful examples). 
The existence proof is essentially based on the Schauder fixed point theorem, which yields a local in time solution. To apply this theorem, we need to prove the compactness of the fixed point operator (acting on both unknowns $\vartheta$ and $\chi$ ), which is reached by parabolic regularizing effects for $\vartheta$ and through the compactness of $\mathcal{J}$ and the Ascoli theorem for $\chi$. Indeed, the only spatial regularization effect of (1.2) is due to the presence of $\mathcal{J}$. We remark that the argument used here is completely different with respect to the one devised in [12], where the first-order analog of (1.2) was studied by parabolic regularization. Indeed, when $\varepsilon>0$, adding a Laplace operator would not help at all in the proof of the existence theorem due to the quoted difficulties in handling hyperbolic PDEs with singular potentials (see [19]). In this regard, our method of treating (1.2) with ODE techniques seems to be more natural. After proving the global existence, uniqueness is obtained via standard methods. However, also at this stage it seems necessary to work within an $L^{\infty}$ framework rather than with energy bounded solutions.

In addition to the well-posedness, we analyze the long time behavior of the solution, i.e., its $\omega$-limit set. Our starting point is the investigation performed in [12] in the firstorder case $(\varepsilon=0)$. If the potential $W$ is convex and the source $f$ suitably tends to 0 as $t \nearrow \infty$, we show that any trajectory provided by the existence theorem admits a nonempty $\omega$-limit, which only contains pairs $\left(\vartheta_{\infty}, \chi_{\infty}\right)$ where $\vartheta_{\infty} \equiv 0$ and $\chi_{\infty}$ is a solution to the stationary problem

$$
W^{\prime}(v)=\mathcal{J}[v], \quad \text { in } \Omega .
$$

Furthermore, in case $W$ is real analytic, using the nonsmooth version [12, Thm. 5.1] of the Łojasiewicz-Simon inequality (see, for instance, $[9,11,25,29,30,31]$ ), we are able to show that the $\omega$-limit reduces to a singleton. Then, following the methods developed in $[10,24]$ and refined in [23] for the long time analysis of asymptotically autonomous PDEs, we also prove that, if the $L^{2}$-norm of $f(t)$ decays to 0 as $t$ goes to $\infty$ with a prescribed rate, then also the convergence rate of the $L^{2}$-norm of $\chi(t)-\chi_{\infty}$ can be quantitatively estimated.

In order to motivate the convexity assumption on $W$, we remark that, due to the lack of spatial regularization effects in (1.2), a strong convergence of $\chi(t)$ to $\chi_{\infty}$ is not immediate to prove. Also, the passage to the limit has to exploit somehow the compactness of $\mathcal{J}$. In fact, comparing terms in (1.9), we deduce that this property is basic to establishing the strong convergence of $W^{\prime}(\chi(t))$ to its limit, which can be then identified with $W^{\prime}\left(\chi_{\infty}\right)$ by using classical monotonicity tools like, e.g., [2, Prop. 1.1, p. 42], provided that $W^{\prime}$ is increasing, i.e., $W$ is convex. Understanding the structure of the $\omega$-limit (i.e., checking whether it contains only solutions of (1.9)) in case $W$ is not convex is an open (and, we think, hard) problem, even in the first-order case analyzed in [12]. We conclude by pointing out that, differently from the standard diffusive case, even for convex $W$ equation (1.9) may have infinite solutions provided that $d>1$ (see the example described in [12, Sec. 1]). Therefore, it is a nontrivial fact to establish that the $\omega$-limit of a (smooth) solution contains only a single point.

The paper is organized as follows. In the next section, we introduce some notation, we provide a list of hypotheses, and we state our main results. Well-posedness is then shown in Section 3 by means of an approximation procedure combined with a fixed point 
argument. Finally, Section 4 is devoted to the analysis of the long time behavior and the proof of the decay estimates.

2. Main results. Let us start by introducing some notation. Let $H:=L^{2}(\Omega)$ and $V:=H_{0}^{1}(\Omega)$. Denote by $(\cdot, \cdot)$ the scalar product in $H$ and by $\|\cdot\|$ the induced norm. In general, $\|\cdot\|_{X}$ will indicate the norm in a generic real Banach space $X$. Let $V$ be endowed with the norm $\|\cdot\|_{V}:=\|\nabla \cdot\|$. Let us identify $H$ with its topological dual $H^{\prime}$ so that we have the continuous and compact inclusions $V \subset H \subset V^{\prime}$.

Regarding the potential $W$ we suppose

$$
\begin{gathered}
W \in C^{2}\left((-1,1) ; \mathbb{R}^{+}\right), \quad \lim _{r \rightarrow 1^{-},-1^{+}} W(r)=+\infty, \\
\exists \lambda \in \mathbb{R}: W^{\prime \prime}(r) \geq-\lambda, \quad \forall r \in(-1,1) .
\end{gathered}
$$

It is easy to see that (W1)-(W2) entail

$$
W^{\prime}(s) s \geq W(s)-\frac{\lambda s^{2}}{2}-W(0), \quad \forall s \in(-1,1) .
$$

Moreover, if $u \in L^{\infty}(\Omega)$ is any function such that $W(u) \in L^{\infty}(\Omega)$, then

$$
\exists \delta=\delta\left(W,\|u\|_{L^{\infty}(\Omega)}\right) \quad \text { such that }-1+\delta \leq u(x) \leq 1-\delta \quad \text { for a.e. } x \in \Omega .
$$

Consider now the operator $\mathcal{J}$. On account of [12], we require the following:

$$
\mathcal{J} \in \mathcal{L}(H ; H) \text { is self-adjoint and compact, }
$$

$$
\mathcal{J} \text { is compact from } L^{\infty}(\Omega) \text { to } C(\bar{\Omega}) \text {. }
$$

Since in the sequel we have to analyze equation (1.1) in an $L^{p}$-setting, we recall some basic results. For $p \in(1, \infty)$ and $T>0$, we set

$$
\mathcal{X}_{p}=\mathcal{X}_{p}(T):=L^{p}\left(0, T ; D\left(A_{p}\right)\right) \cap W^{1, p}\left(0, T ; L^{p}(\Omega)\right),
$$

where $A_{p}:=-\Delta: D\left(A_{p}\right)=W^{2, p}(\Omega) \cap W_{0}^{1, p}(\Omega) \rightarrow L^{p}(\Omega)$. When $p=2$, we simply set $A:=A_{2}$. The space $\mathcal{X}_{p}$ is endowed with the natural (Banach) norm and it is known (see, e.g., [1, Thm. 4.10.2, p. 181]) that the continuous embedding

$$
\mathcal{X}_{p} \subset \operatorname{BUC}\left([0, T] ; \mathcal{D}_{p}(\Omega)\right)
$$

holds, where $\mathcal{D}_{p}(\Omega)$ stands for the interpolation space $\left(L^{p}(\Omega), D\left(A_{p}\right)\right)_{1-1 / p, p}$. For instance, for $p \geq 2, \mathcal{D}_{p}(\Omega)$ coincides with $W^{2-\frac{2}{p}, p}(\Omega) \cap W_{0}^{1, p}(\Omega)$. We now recall the theorem (see, e.g., [1, Thm. 4.10.7, p. 184]):

Theorem 2.1. Let $p \in(1, \infty), T>0$, and take

$$
u_{0} \in \mathcal{D}_{p}(\Omega), \quad g \in L^{p}\left(0, T ; L^{p}(\Omega)\right) .
$$

Then, the solution $u$ defined on $(0, T)$ to the problem

$$
u_{t}+A_{p} u=g, \quad u_{\mid t=0}=u_{0},
$$

lies in $\mathcal{X}_{p}(T)$. Moreover, there exists $c>0$ independent of $T$ and such that

$$
\|u\|_{\mathcal{X}_{p}(T)} \leq c\left(\|g\|_{L^{p}\left(0, T ; L^{p}(\Omega)\right)}+\left\|u_{0}\right\|_{\mathcal{D}_{p}(\Omega)}\right) .
$$


We now formulate our problem (see (1.1)-(1.2)) as

$$
\begin{aligned}
& \vartheta_{t}+\chi_{t}+A \vartheta=f, \quad \text { a.e. in } \Omega \times(0, T), \\
& \varepsilon \chi_{t t}+\chi_{t}+W^{\prime}(\chi)=\mathcal{J}[\chi]+\vartheta, \quad \text { a.e. in } \Omega \times(0, T), \\
& \vartheta_{\mid t=0}=\vartheta_{0}, \quad \text { in } \Omega, \\
& \chi_{\mid t=0}=\chi_{0}, \quad\left(\chi_{t}\right)_{\mid t=0}=\chi_{1}, \quad \text { a.e. in } \Omega,
\end{aligned}
$$

where

$$
\vartheta_{0} \in V
$$

and

$$
f \in L^{2}(0, T ; H)
$$

Moreover, we suppose

$$
\chi_{0}, \chi_{1} \in L^{\infty}(\Omega), \quad \exists \delta_{0}>0:-1+\delta_{0} \leq \chi_{0} \leq 1-\delta_{0} \quad \text { a.e. in } \Omega
$$

where the latter property is equivalent to saying that $W\left(\chi_{0}\right) \in L^{\infty}(\Omega)$.

Our well-posedness theorem reads

Theorem 2.2. Let (W1)-(W2), (J1)-(J2), (init $\vartheta)-($ init $\chi),(f 1)$ hold. Then, for any given $T>0$, there exists one and only one pair $(\vartheta, \chi)$, with

$$
\begin{gathered}
\vartheta \in \mathcal{X}_{2}(T) \cap C^{0}([0, T] ; V), \\
\chi, \chi_{t}, W(\chi) \in L^{\infty}(\Omega \times(0, T)), \quad \chi_{t t} \in L^{2}\left(0, T ; L^{\infty}(\Omega)\right),
\end{gathered}
$$

that solves (2.8)-(2.11). Moreover, for a.a. $t \in(0, T),(2.2)$ holds with $u(\cdot):=\chi(\cdot, t)$. Next, given two triplets $\left(\vartheta_{0,1}, \chi_{0,1}, \chi_{1,1}\right),\left(\vartheta_{0,2}, \chi_{0,2}, \chi_{1,2}\right)$ of initial data satisfying conditions (init $\vartheta)-\left(\right.$ init $\chi$ ) (the latter w.r.t. possibly different constants $\delta_{0 i}>0, i=1,2$ ) and denoting the corresponding solutions by $\left(\vartheta_{1}, \chi_{1}\right),\left(\vartheta_{2}, \chi_{2}\right)$, we have the continuous dependence estimate

$$
\begin{aligned}
& \left\|\left(\vartheta_{1}-\vartheta_{2}\right)(t)\right\|+\left\|\nabla\left(\vartheta_{1}-\vartheta_{2}\right)\right\|_{L^{2}(0, t ; H)}+\sqrt{\varepsilon}\left\|\left(\chi_{1}-\chi_{2}\right)_{t}(t)\right\|+\left\|\left(\chi_{1}-\chi_{2}\right)(t)\right\| \\
& \quad \leq \Lambda\left(\left\|\vartheta_{0,1}-\vartheta_{0,2}\right\|+\sqrt{\varepsilon}\left\|\chi_{1,1}-\chi_{1,2}\right\|+\left\|\chi_{0,1}-\chi_{0,2}\right\|\right), \quad \forall t \in[0, T],
\end{aligned}
$$

where the positive constant $\Lambda$ depends on $T, \Omega, W, \mathcal{J}, f$ and on the initial data (in particular, on $\left.\delta_{0 i}, i=1,2\right)$, but it does not depend on $\varepsilon$.

REMARK 2.3. The second assumption of (W1) does not cover, for instance, the case of a potential which is bounded in $[-1,1]$. A typical case is the logarithmic potential (cf., e.g., $[22,26]$ )

$$
W(r)=(1+r) \log (1+r)+(1-r) \log (1-r)-\frac{\lambda}{2} r^{2}, \quad r \in(-1,1),
$$

where $\lambda \in \mathbb{R}$. In order to handle potentials of this kind when $\chi$ is ruled by a second-order dynamics, some restrictions seem to be necessary (for details, see Remark 3.3 below). 
We now consider the long time behavior. The main tool we shall use to prove that the $\omega$-limit is a singleton is the following nonsmooth version of the Eojasiewicz-Simon inequality (see [12, Thm. 5.1]). For the sake of clarity, we report the precise statement, which in our notation takes the form

Theorem 2.4. Let (W1)-(W2) and (J1)-(J2) hold. In addition, suppose that

$$
W \text { is real analytic on }(-1,1) \text {, }
$$

and define $F: H \rightarrow \mathbb{R}$ by

$$
F(v):=-\frac{1}{2}(\mathcal{J}[v], v)+(W(v), 1),
$$

for any $v \in H$. If $\chi_{*} \in C(\bar{\Omega})$ is a critical point for $F$, then there exist $\rho \in(0,1 / 2), \ell>0$ and $\sigma>0$ such that

$$
\left|F(v)-F\left(\chi_{*}\right)\right|^{1-\rho} \leq \ell\left\|-\mathcal{J}[v]+W^{\prime}(v)\right\|,
$$

for all $v \in H$ such that $\left\|v-\chi_{*}\right\| \leq \sigma$.

REMARK 2.5. Assumption (2.16) can be slightly weakened (see [11]). Indeed, using assumptions (W1), (J1) and a simple maximum principle argument, it is easy to prove that any solution $\chi_{*}$ to (1.9) satisfies a separation property of the form

$$
-1+\delta_{1} \leq \chi_{*}(x) \leq 1-\delta_{1}, \quad \text { for all } x \in \Omega,
$$

where $\delta_{1}$ depends only on the expression of $W$ and on $\|\mathcal{J}\|_{\mathcal{L}(H ; H)}$. Then, it suffices that $W$ is real analytic on an open interval containing $\left[-1+\delta_{1}, 1-\delta_{1}\right]$.

The result about the long time behavior of the solutions is

Theorem 2.6. Let (W1)-(W2), (J1)-(J2), (init $\vartheta)-($ init $\chi)$, (f1) hold. In addition, let

$$
f \in L^{2}(0, \infty ; H) \text {. }
$$

Then, the solution $(\vartheta, \chi)$ given by Theorem 2.2 satisfies, as $t$ goes to $\infty$,

$$
\begin{gathered}
\vartheta(t) \rightarrow 0 \quad \text { strongly in } V, \\
\chi_{t}(t), \sqrt{\varepsilon} \chi_{t t}(t) \rightarrow 0 \text { strongly in } H .
\end{gathered}
$$

If $W$ is convex (i.e., $\lambda=0$ in (W2)), then $(\vartheta, \chi)$ admits a nonempty $\omega$-limit set which consists of pairs $\left(0, \chi_{\infty}\right)$, where $\chi_{\infty}$ satisfies $(1.9)$. More precisely, if $\left(0, \chi_{\infty}\right)$ is an element of the $\omega$-limit and $t \nearrow \infty$, we have

$$
\text { a subsequence of } \chi(t) \rightarrow \chi_{\infty} \text { weakly in } H,
$$

where the convergence (2.22) is strong if (W2) holds for some $\lambda<0$. If there exists $\mu>0$ such that

$$
\sup _{t \geq 0} t^{1+\mu} \int_{t}^{\infty}\|f(s)\|^{2} \mathrm{~d} s<\infty
$$

and if $W$ is strictly convex (i.e., $\lambda<0$ in (W2)) and satisfies (2.16), then the $\omega$-limit reduces to $\left\{\left(0, \chi_{\infty}\right)\right\}$ and $\chi(t)$ converges to $\chi_{\infty}$ in $H$ as $t$ goes to $\infty$. Besides, if

$$
\mu \geq \frac{2 \rho}{1-2 \rho}
$$


one can find $t^{*}=t^{*}(\varepsilon)>0$ and $c>0$ independent of $\varepsilon$ such that

$$
\left\|\chi(t)-\chi_{\infty}\right\| \leq c t^{-\frac{\rho}{1-2 \rho}}, \quad \text { for all } t \geq t^{*} .
$$

Otherwise, for any $\rho_{0} \in(0, \rho)$ such that

$$
\mu \geq \frac{2 \rho_{0}}{1-2 \rho_{0}}
$$

one can find $t^{* *}=t^{* *}(\varepsilon)>0$ and $c>0$ independent of $\varepsilon$ such that

$$
\left\|\chi(t)-\chi_{\infty}\right\| \leq c t^{-\frac{\rho_{0}}{1-2 \rho_{0}}}, \quad \text { for all } t \geq t^{* *} .
$$

REMARK 2.7. By analyzing the following proofs, it is not difficult to realize that they can be easily adapted to the case $\varepsilon=0$ (compare with [12], where a different approach is used). Consequently, Theorems 2.2 and 2.6 can be rephrased for the case $\varepsilon=0$.

3. Proof of Theorem 2.2. We start proving the existence of a local (in time) solution to our problem by means of the Schauder fixed point theorem. In this argument, we need to work with Hölder continuous solutions to (1.1) and continuous solutions to (1.2). Thus, we have to approximate the initial data in the following way. Regarding $\vartheta_{0}$ and $f$, on account of Theorem 2.1, we will assume

$$
\vartheta_{0} \in \mathcal{D}_{q}(\Omega), \quad f \in L^{q}\left(0, T ; L^{q}(\Omega)\right),
$$

with $q \in(2, \infty)$ large enough to have the inclusion

$$
\mathcal{X}_{q}(T) \subset C^{0, \alpha}\left([0, T] ; C^{0, \alpha}(\bar{\Omega})\right),
$$

with compact embedding, for some $\alpha>0$. Here we recall that the admissible exponents $q$ depend, of course, on the space dimension. For instance, if $d=3, q$ can be any value strictly greater than $\frac{5}{2}$ as can be shown by standard interpolation tools. We also observe that the embedding constant in (3.2) can be taken independent of $T$ on bounded time intervals; in other words, it does not explode as $T \searrow 0$ (while it does explode as $T \nearrow \infty$ ). As far as the initial values for $\chi$ are concerned, in addition to (init $\chi$ ), we will suppose

$$
\chi_{0}, \chi_{1} \in C(\bar{\Omega}) .
$$

Let us now set, for $t>0, Q_{t}:=\Omega \times(0, t)$, and define $B=B(R, T)$ as

$$
\begin{aligned}
B:= & \left\{(u, v) \in C\left([0, T] ; C^{0, \alpha}(\bar{\Omega})\right) \times C\left(\overline{Q_{T}}\right):\right. \\
& \left.\|u\|_{C\left([0, T] ; C^{0, \alpha}(\bar{\Omega})\right)} \leq R,\|v\|_{C\left(\overline{Q_{T}}\right)} \leq 1, u_{\mid t=0}=\vartheta_{0}, v_{\mid t=0}=\chi_{0}\right\},
\end{aligned}
$$

where $R$ is a positive number such that

$$
R \geq 2\left\|\vartheta_{0}\right\|_{C^{0, \alpha}(\bar{\Omega})}
$$

Observe that $B$ is a nonempty convex, closed set with respect to the natural (Banach) topology. In order to introduce the fixed point procedure, we assume that $(\bar{\vartheta}, \bar{\chi}) \in B$ is 
"frozen" on the right hand side of (2.9). Then the fixed point map is constructed in the following

Lemma 3.1. Let (W1)-(W2), (J1)-(J2), (init $\chi),(3.1)$, and (3.3) hold. Let $R, T>0$ be fixed and let $B$ be defined as in (3.4). For any given $(\bar{\vartheta}, \bar{\chi}) \in B$, there exists a unique pair $(\vartheta, \chi): Q_{T} \rightarrow \mathbb{R}^{2}$ such that

$$
\begin{aligned}
& \vartheta \in \mathcal{X}_{q}(T), \\
& \chi, \chi_{t}, \chi_{t t} \in C\left(\overline{Q_{T}}\right),
\end{aligned}
$$

which solves

$$
\begin{aligned}
& \vartheta_{t}+\chi_{t}+A \vartheta=f, \\
& \varepsilon \chi_{t t}+\chi_{t}+W^{\prime}(\chi)=\mathcal{J}[\bar{\chi}]+\bar{\vartheta}
\end{aligned}
$$

almost everywhere in $Q_{T}$ together with (2.10)-(2.11). Moreover, $\chi$ enjoys the separation property, that is,

$$
\exists \delta=\delta\left(R, \mathcal{J}, W, \delta_{0}\right)>0:-1+\delta \leq \chi(x, t) \leq 1-\delta, \quad \forall(x, t) \in \overline{Q_{T}},
$$

where $\delta$ is independent both of $x \in \Omega$ and of $T$.

Proof. Here and in the sequel of the paper, we shall indicate by $c$ a generic positive constant which may vary even within the same line. This constant may depend on $\Omega$, $\varepsilon, \mathcal{J}, W, f$ and on the initial data, but it is independent of $T$. In any case, any further dependency will be explicitly pointed out. Let us denote by $m=m(x, t)$ the right hand side of (3.9). Thanks to (3.4) and the properties of $\mathcal{J}$, we have that $m \in C\left(\overline{Q_{T}}\right)$. In particular, there exists $M>0$, only depending on $R$ and $\mathcal{J}$, such that

$$
|m(x, t)| \leq M, \quad \forall(x, t) \in \overline{Q_{T}}
$$

Then, fixing $x \in \Omega$, it is clear that the Cauchy problem for the ODE obtained from (3.9) admits one and only one local in time classical solution $\chi$. To show that such a solution is in fact global, one can, e.g., test (3.9) by $\chi_{t}+\eta \chi$, for $\eta>0$ to be chosen, and, still for fixed $x$, integrate in time. Using $(2.1)$, we get

$$
\begin{aligned}
& \frac{\mathrm{d}}{\mathrm{d} t} \Phi+(1-\varepsilon \eta)\left|\chi_{t}\right|^{2}+\eta W(\chi) \\
& \quad \leq M\left|\chi_{t}+\eta \chi\right|+\eta W(0)+\frac{\lambda \eta}{2}|\chi|^{2} \leq \frac{1-\varepsilon \eta}{2}\left|\chi_{t}\right|^{2}+\eta W(0)+c(M, \eta, \lambda)
\end{aligned}
$$

where

$$
\Phi:=\frac{\varepsilon}{2}\left|\chi_{t}\right|^{2}+\frac{\eta}{2}|\chi|^{2}+\varepsilon \eta \chi \chi_{t}+W(\chi)
$$

Then, it is easy to realize that $\eta$ can be chosen in a way independent of $\varepsilon \in(0,1)$ and such that, for some $\kappa>0$,

$$
\frac{\mathrm{d}}{\mathrm{d} t} \Phi(x, t)+\kappa \Phi(x, t) \leq c(M, \lambda), \quad \text { for all }(x, t) \in \overline{Q_{T}}
$$

and

$$
\frac{\varepsilon}{4}\left|\chi_{t}\right|^{2}+\frac{1}{16}|\chi|^{2}+W(\chi) \leq \Phi \leq \varepsilon\left|\chi_{t}\right|^{2}+\frac{1}{4}|\chi|^{2}+W(\chi)
$$


Thus, using the Gronwall lemma in the differential form, we get

$$
\frac{\varepsilon}{4}\left|\chi_{t}(t)\right|^{2}+\frac{1}{16}|\chi(t)|^{2}+W(\chi(t)) \leq\left(\varepsilon\left|\chi_{1}\right|^{2}+\frac{1}{4}\left|\chi_{0}\right|^{2}+W\left(\chi_{0}\right)\right) e^{-\kappa t}+c,
$$

where the dependence on $x$ of all functions is omitted. Thanks to assumption (init $\chi$ ), we then get (3.10) (regarding $\delta$, we stress that $M$ depends only on $R$ and $\mathcal{J}$ ).

At this point, noting that $W^{\prime}(\chi)$ is bounded in $C\left(\overline{Q_{T}}\right)$ by a constant independent of $T$, (init $\chi$ ) and standard regularity and continuous dependence results for ODEs (applied here uniformly with respect to the variable $x$ ) entail (3.7). More precisely, we have

$$
\|\chi\|_{C\left(\overline{Q_{T}}\right)}+\left\|\chi_{t}\right\|_{C\left(\overline{Q_{T}}\right)}+\left\|\chi_{t t}\right\|_{C\left(\overline{Q_{T}}\right)} \leq c .
$$

Dragging now the known value of $\chi_{t}$ into (1.1) and applying Theorem 2.1, we obtain the existence of one and only one function $\vartheta$ satisfying (3.6) and (2.10) and such that

$$
\|\vartheta\|_{\mathcal{X}_{q}(T)} \leq c\left(\left\|f-\chi_{t}\right\|_{L^{q}\left(0, T ; L^{q}(\Omega)\right)}+\left\|\vartheta_{0}\right\|_{\mathcal{D}_{q}(\Omega)}\right) \leq c\left(T^{1 / q}+1\right) .
$$

The proof is thus concluded.

Lemma 3.1 allows us to define the operator $\mathcal{S}: B \rightarrow C\left([0, T] ; C^{0, \alpha}(\bar{\Omega})\right) \times C\left(\overline{Q_{T}}\right)$ by setting

$$
(\vartheta, \chi)=: \mathcal{S}(\bar{\vartheta}, \bar{\chi})=\left(\mathcal{S}_{1}(\bar{\vartheta}, \bar{\chi}), \mathcal{S}_{2}(\bar{\vartheta}, \bar{\chi})\right)
$$

The aim of the next result is to prove the local (in time) existence of a (smooth) solution to our problem by means of the Schauder theorem applied to $\mathcal{S}$.

Lemma 3.2. Let (W1)-(W2), (J1)-(J2), (init $\chi),(3.1)$, and (3.3) hold. Let $R$ be any number fulfilling (3.5). Then, there exists $T_{0}>0$ depending on $f, R, \mathcal{J}, W$ such that problem (2.8)-(2.11) admits at least a solution $(\vartheta, \chi)$ defined for $t \in\left(0, T_{0}\right)$. Moreover, this solution satisfies

$$
\begin{aligned}
& \vartheta \in \mathcal{X}_{q}\left(T_{0}\right), \\
& \chi, \quad \chi_{t}, \quad \chi_{t t} \in C\left(\overline{Q_{T_{0}}}\right),
\end{aligned}
$$

and the component $\chi$ fulfills (3.10).

Proof. Let us first prove that, for a suitable $T_{0}>0, \mathcal{S}$ maps $B\left(R, T_{0}\right)$ into itself. As far as $\chi$ is concerned, it is easy to see that everything works thanks to (3.7), (2.11) and (3.10). Considering the component $\vartheta$, by (3.18), (3.2), and (3.5), we have that

$$
\|\vartheta(t)\|_{C^{0, \alpha}(\bar{\Omega})} \leq\left\|\vartheta(t)-\vartheta_{0}\right\|_{C^{0, \alpha}(\bar{\Omega})}+\left\|\vartheta_{0}\right\|_{C^{0, \alpha}(\bar{\Omega})} \leq c T^{\alpha}\left(T^{1 / q}+1\right)+R / 2 .
$$

Thus, we can choose $T=T_{0}$ so small such that $\mathcal{S}\left(B\left(R, T_{0}\right)\right) \subseteq B\left(R, T_{0}\right)$. For simplicity we set again $B=B\left(R, T_{0}\right)$. The proof will now be achieved by means of the Schauder fixed point theorem. Let us prove first that $\mathcal{S}(B)$ is relatively compact with respect to the topology of $B$. On account of estimate (3.18) and compactness of the embedding (3.2), the relative compactness of $\mathcal{S}_{1}(B)$ in $C^{0, \alpha}\left(\left[0, T_{0}\right] ; C^{0, \alpha}(\bar{\Omega})\right)$ holds. As far as $\mathcal{S}_{2}$ is concerned, let us show that the Ascoli theorem can be applied to the set $\mathcal{S}_{2}(B)$ yielding relative compactness in $C\left(\overline{Q_{T_{0}}}\right)$. By definition of $B$ and estimates (3.17), the equiboundedness of $\mathcal{S}_{2}(B)$ follows. The proof of equicontinuity is slightly more difficult. We start noting that, by (3.4), the properties of $\mathcal{J}$, and the compact embedding $C^{0, \alpha}(\bar{\Omega}) \subset C(\bar{\Omega})$, there exists a set $K$ compactly embedded into $C(\bar{\Omega})$ and 
such that $m(\cdot, t):=\bar{\vartheta}(\cdot, t)+\mathcal{J}[\bar{\chi}](\cdot, t) \in K$ for all $t \in\left[0, T_{0}\right]$ and $(\bar{\vartheta}, \bar{\chi}) \in B$. Letting $(\vartheta, \chi)=\mathcal{S}(\bar{\vartheta}, \bar{\chi})$, for $(x, t),(y, s) \in \overline{Q_{T_{0}}}$, we have

$$
|\chi(x, t)-\chi(y, s)| \leq|\chi(x, t)-\chi(y, t)|+|\chi(y, t)-\chi(y, s)| .
$$

Thus, we have to show that, as $|(x, t)-(y, s)| \rightarrow 0$, the right hand side goes to 0 uniformly with respect to the choice of $(\bar{\vartheta}, \bar{\chi}) \in B$. Actually, the second term can be handled easily, owing to (3.17). Instead, to deal with the first term, let us set, for $t \in\left[0, T_{0}\right]$ and $i=0,1$,

$$
\widetilde{m}(t):=m(x, t)-m(y, t), \quad \tilde{\chi}(t):=\chi(x, t)-\chi(y, t), \quad \tilde{\chi}_{i}:=\chi_{i}(x)-\chi_{i}(y) .
$$

Then, consider equation (3.9) (whose right hand side, we recall, has been noted as $m$ ) at the space points $x$ and $y$, take the difference, and test it by $\tilde{\chi}_{t}+\eta \tilde{\chi}$, for some $\eta>0$. Using the uniform bound on $W(\chi)$, it is not difficult to obtain

$$
\frac{\mathrm{d}}{\mathrm{d} t}\left[\frac{\varepsilon}{2}\left|\widetilde{\chi}_{t}\right|^{2}+\frac{\eta}{2}|\widetilde{\chi}|^{2}+\varepsilon \eta \tilde{\chi} \widetilde{\chi}_{t}\right] \leq c\left(|\widetilde{m}|^{2}+|\widetilde{\chi}|^{2}+\left|\widetilde{\chi}_{t}\right|^{2}\right)
$$

whence, by the Gronwall lemma, for $t \in\left[0, T_{0}\right]$ we get

$$
\varepsilon\left|\widetilde{\chi}_{t}(t)\right|^{2}+\eta|\widetilde{\chi}(t)|^{2} \leq c\left(T_{0}\right)\left(\left|\widetilde{\chi}_{1}\right|^{2}+\left|\widetilde{\chi}_{0}\right|^{2}+\int_{0}^{t}|\widetilde{m}(s)|^{2} \mathrm{~d} s\right) .
$$

Using (uniform) continuity of $\chi_{0}$ and $\chi_{1}$ (cf. (init $\left.\chi\right)$ ) and equicontinuity of the functions $m(\cdot, s)$ with respect to $s \in\left[0, T_{0}\right]$ (remember that they take values in the compact set $K$ ), it is then easy to conclude the equicontinuity of $\mathcal{S}_{2}(B)$, as desired. It remains to demonstrate that $\mathcal{S}$ is a continuous mapping. Letting $\left\{\left(\bar{\vartheta}_{n}, \bar{\chi}_{n}\right)\right\}$ be a sequence in $B$ converging to an element $(\bar{\vartheta}, \bar{\chi})$, by compactness of $\mathcal{S}$ we can extract a subsequence (not relabelled) of $\left\{\left(\vartheta_{n}, \chi_{n}\right)\right\}=\left\{\mathcal{S}\left(\bar{\vartheta}_{n}, \bar{\chi}_{n}\right)\right\} \subset B$ converging to some $(\vartheta, \chi) \in B$. Writing (3.9) for the index $n$ and using the uniform convergence of $\left\{\chi_{n}\right\}$ (holding by compactness of $\mathcal{S}_{2}$ ) in $\overline{Q_{T_{0}}}$ and the separation property (3.10) (which holds uniformly in $n$ ), it is apparent that we can pass to the limit in the nonlinear term $W^{\prime}(\cdot)$, so that the limit equation $(3.9)$ holds for $(\vartheta, \chi)$ and $(\bar{\vartheta}, \bar{\chi})$. Next, the passage to the limit in (3.8) is trivial due to the linearity. Finally, thanks to the uniqueness property stated in Lemma 3.1, convergence to $(\vartheta, \chi)$ holds for the whole sequence $\left\{\left(\vartheta_{n}, \chi_{n}\right)\right\}$. This clearly entails continuity of $\mathcal{S}$. Therefore $\mathcal{S}$ has a fixed point in $B$ and this ends the proof.

Existence. We can now complete the proof of existence. Namely, we need to extend the local solution up to any fixed time $T>0$ and remove the regularity assumptions (3.1) and (3.3). Take $\vartheta_{0}$ and $f$ satisfying (init $\vartheta$ ) and (f1), respectively. Then, consider two approximating sequences $\left\{\vartheta_{0, n}\right\} \subset \mathcal{D}_{q}(\Omega)$ and $\left\{f_{n}\right\} \subset L^{q}\left(0, T ; L^{q}(\Omega)\right)$ such that, as $n$ goes to $\infty$,

$$
\vartheta_{0, n} \rightarrow \vartheta_{0} \text { strongly in } V, \quad f_{n} \rightarrow f \text { strongly in } L^{2}(0, T ; H) .
$$

Moreover, consider $\chi_{0}, \chi_{1}$ satisfying only (init $\chi$ ) and take an approximating sequence $\left\{\left(\chi_{0, n}, \chi_{1, n}\right)\right\} \subset C(\bar{\Omega}) \times C(\bar{\Omega})$, bounded in $L^{\infty}(\Omega) \times L^{\infty}(\Omega)$ and such that

$$
\left(\chi_{0, n}, \chi_{1, n}\right) \rightarrow\left(\chi_{0}, \chi_{1}\right) \text { strongly in } H \times H .
$$

The above sequences can be explicitly constructed by standard mollification. Let us denote by $\left(\vartheta_{n}, \chi_{n}\right)$ a sequence of (local) in time solutions corresponding to the initial data $\left(\vartheta_{0, n}, \chi_{0, n}, \chi_{1, n}\right)$ and to the source $f_{n}$. Their final times $T_{0, n}$ are yielded by Lemma 3.2 
and may depend on $n$. Nevertheless, in what follows we shall prove some a priori estimates independent of $n$ in order to remove both the approximation and the restriction on the final time. Thus, let $T>0$ be arbitrary and let us show that the limit of $\left(\vartheta_{n}, \chi_{n}\right)$ can be extended up to $T$. For simplicity, we shall write the forthcoming estimates for the approximating solutions by referring directly to times $t \leq T$. In the sequel $C$ will indicate a generic positive constant, similar to $c$ (see above), which however may depend on $T$ (but not on $n$ ). In addition, from this point on, we shall assume both $c$ and $C$ independent of $\varepsilon$.

As a first estimate, we multiply $(2.8)$ by $\vartheta_{n}$ and $(2.9)$ by $\left(\chi_{n}\right)_{t}$ (both equations are here intended for the $n$-approximation). Adding the resulting identities together, integrating over $Q_{t}$, and using (J1), (f1), we obtain

$$
\frac{\mathrm{d}}{\mathrm{d} t} G+\left\|\nabla \vartheta_{n}\right\|^{2}+\left\|\chi_{n, t}\right\|^{2} \leq\left(\vartheta_{n}, f_{n}\right)
$$

where

$$
G:=\frac{1}{2}\left\|\vartheta_{n}\right\|^{2}+\frac{\varepsilon}{2}\left\|\chi_{n, t}\right\|^{2}-\frac{1}{2}\left(\mathcal{J}\left[\chi_{n}\right], \chi_{n}\right)+\left(W\left(\chi_{n}\right), 1\right) .
$$

The functional $G$ is bounded from below by (W1) and (J1). Then, using the Young and the Poincare inequalities on the right hand side, we easily get the bounds

$$
\begin{aligned}
& \left\|\vartheta_{n}\right\|_{L^{\infty}(0, T ; H)}+\left\|\vartheta_{n}\right\|_{L^{2}(0, T ; V)} \leq C, \\
& \varepsilon^{1 / 2}\left\|\chi_{n, t}\right\|_{L^{\infty}(0, T ; H)}+\left\|\chi_{n, t}\right\|_{L^{2}(0, T ; H)} \leq C, \\
& \left\|W\left(\chi_{n}\right)\right\|_{L^{\infty}\left(0, T ; L^{1}(\Omega)\right)} \leq C .
\end{aligned}
$$

In particular, (3.30) entails that $-1 \leq \chi_{n}(x, t) \leq 1$ almost everywhere in $Q_{T}$. Bound (3.28) and the standard Hilbert theory applied to (2.8) yield

$$
\left\|\vartheta_{n}\right\|_{H^{1}(0, T ; H)}+\left\|\vartheta_{n}\right\|_{C^{0}([0, T] ; V)}+\left\|\vartheta_{n}\right\|_{L^{2}\left(0, T ; H^{2}(\Omega)\right)} \leq C .
$$

Then, let us test $(2.9)$ by $\left(\chi_{n}\right)_{t}+\eta \chi_{n}$, for some $\eta>0$ to be chosen properly. Here, we do not integrate over $\Omega$. Adding the inequality $\left|\chi_{n}\right|^{2} \leq 1$, noting that there exists $M>0$ such that $\mathcal{J}\left[\chi_{n}\right] \leq M$ for almost every $(x, t) \in Q_{T}$, and using (2.1), the continuous embedding $H^{2}(\Omega) \subset L^{\infty}(\Omega)$, and the elementary Young inequality, we get

$$
\begin{aligned}
& \frac{\mathrm{d}}{\mathrm{d} t}\left[\frac{\varepsilon}{2}\left|\chi_{n, t}\right|^{2}+\frac{\eta}{2}\left|\chi_{n}\right|^{2}+\varepsilon \eta \chi_{n} \chi_{n, t}+W\left(\chi_{n}\right)\right]+(1-\varepsilon \eta)\left|\chi_{n, t}\right|^{2}+\eta W\left(\chi_{n}\right)+\left|\chi_{n}\right|^{2} \\
& \quad \leq \frac{1}{4}\left|\chi_{n, t}\right|^{2}+C(M, \eta)+c\left\|\vartheta_{n}\right\|_{H^{2}(\Omega)}^{2},
\end{aligned}
$$

where all terms (but the latter) are evaluated at a generic $(x, t) \in Q_{T}$, while the latter is evaluated at the (same) time $t \leq T$. Choosing now $\eta$ small enough and denoting by $\Phi_{n}=\Phi_{n}(x, t)$ the function in square brackets on the left hand side, it is easy to see that

$$
\frac{\mathrm{d}}{\mathrm{d} t} \Phi_{n}(x, t)+\kappa \Phi_{n}(x, t) \leq C(M)+c\left\|\vartheta_{n}(t)\right\|_{H^{2}(\Omega)}^{2},
$$

for all $(x, t) \in Q_{T}$ and some $\kappa>0$ independent of $\varepsilon \in(0,1)$. Here $\eta$ has been chosen so small that $\Phi_{n}$ satisfies a relation analogous to (3.15). Applying now a generalized form 
of the differential Gronwall lemma (cf., e.g., [18, Lemma 2.5]) to (3.33), we deduce

$$
\Phi_{n}(x, t) \leq \Phi_{n}(x, 0) e^{-\kappa t}+\frac{2 C(M)}{\kappa}+2 c \int_{0}^{t}\left\|\vartheta_{n}(s)\right\|_{H^{2}(\Omega)}^{2} \mathrm{~d} s \leq C,
$$

for all $(x, t) \in Q_{T}$, where in the last inequality we exploited the boundedness (init $\chi$ ) and the third estimate in (3.31). Using (3.15) (written for $\Phi_{n}$ ) and taking the (essential) supremum with respect to $(x, t) \in Q_{T}$ on the left hand side of (3.34), we readily get

$$
\left\|\chi_{n}\right\|_{L^{\infty}\left(Q_{T}\right)}+\varepsilon^{1 / 2}\left\|\chi_{n, t}\right\|_{L^{\infty}\left(Q_{T}\right)}+\left\|W\left(\chi_{n}\right)\right\|_{L^{\infty}\left(Q_{T}\right)} \leq C .
$$

REMARK 3.3. We notice that the latter bound and (W1) entail an $L^{\infty}$ estimate also for the functions $W^{\prime}\left(\chi_{n}\right)$ and $W^{\prime \prime}\left(\chi_{n}\right)$. This is no longer true if the latter condition in (W1) is not satisfied (as for the potential (2.15)). Nevertheless, as will be seen in the uniform estimates below (cf., in particular, (4.5)), the constant $C$ in (3.35) is computable and depends only on $\vartheta_{0}, \chi_{0}, \chi_{1}, W\left(\chi_{0}\right), f$, and $\mathcal{J}$. If these data (and consequently $C$ in (3.35) and $c$ in (4.3) below) are sufficiently small, then clearly the separation property (2.2) and the $L^{\infty}$ bounds on $W^{\prime}\left(\chi_{n}\right)$ and $W^{\prime \prime}\left(\chi_{n}\right)$ still hold. This means that, in fact, if we drop the latter of (W1), then we need to require some compatibility relation between the "smallness" of the above data and the "coercivity" of $W$. On the other hand, on account of [19], we guess that a more careful analysis should show that potentials like (2.15) can be handled, provided that $\varepsilon$ is sufficiently small. Indeed, in this case, a solution to our problem (2.8)-(2.11) should be "close" to the corresponding one with $\varepsilon=0$, for which a comparison argument can be used (see, e.g., [27]).

Finally, a comparison in (2.9), the continuous embedding $H^{2}(\Omega) \subset L^{\infty}(\Omega)$, and (3.28)(3.29) yield

$$
\varepsilon\left\|\chi_{n, t t}\right\|_{L^{2}(0, T ; H)} \leq C .
$$

We are now in a position to pass to the limit as $n \nearrow \infty$. First, we observe that, from (3.28)-(3.29), (3.31), (3.35), (3.36), some weak or weak star convergence properties to suitable limits $(\vartheta, \chi)$ can be deduced at least for subsequences of $\left(\vartheta_{n}, \chi_{n}\right)$ (they will actually hold for the whole sequence thanks to the uniqueness property proved below). Moreover, from (3.30) we get that $W^{\prime}\left(\chi_{n}\right)$ tends to some $w$ weakly star in $L^{\infty}\left(Q_{T}\right)$. At this point, thanks also to (J1), it is easy to pass to the limit both in (2.8) and in (2.9). We only need to check that it is $w=W^{\prime}(\chi)$ almost everywhere in $Q_{T}$. To prove this, we first note that, by (3.35),

$$
\chi_{n}(t) \rightarrow \chi(t) \quad \text { weakly in } H \text { for all } t \in[0, T] .
$$

On the other hand, by (J1) and the Lebesgue theorem, it follows that

$$
\mathcal{J}\left[\chi_{n}\right] \rightarrow \mathcal{J}[\chi] \quad \text { strongly in } L^{2}(0, T ; H)
$$

whence the sequence $m_{n}$ of the right hand sides of (2.9) tends to $m=\mathcal{J}[\chi]+\vartheta$ strongly in $L^{2}(0, T ; H)$. Let us now set, for $n_{1}, n_{2}>0$,

$$
\widetilde{m}:=m_{n_{1}}-m_{n_{2}}, \quad \tilde{\chi}:=\chi_{n_{1}}-\chi_{n_{2}},
$$


write $(2.9)$ for $n_{1}$ and $n_{2}$, take the difference, test it by $\widetilde{\chi}_{t}$, and integrate over $Q_{t}$ for $t \in(0, T]$. Then, adding to the result the trivial relation

$$
\|\tilde{\chi}(t)\|^{2} \leq\left\|\chi_{0, n_{1}}-\chi_{0, n_{2}}\right\|^{2}+\frac{1}{4}\left\|\tilde{\chi}_{t}\right\|_{L^{2}(0, t ; H)}^{2}+4\|\widetilde{\chi}\|_{L^{2}(0, t ; H)}^{2} \quad \text { for } t \in[0, T]
$$

and splitting the $W^{\prime}$-term by Hölder's inequality (note that $W^{\prime}$ can be regarded as a Lipschitz continuous function thanks to (3.35) which entails uniformly in $n$ and $\varepsilon$ the separation property), it is not difficult to obtain, for $t \in[0, T]$,

$$
\frac{\varepsilon}{2}\left\|\widetilde{\chi}_{t}(t)\right\|^{2}+\|\widetilde{\chi}(t)\|^{2} \leq 2\left\|\chi_{0, n_{1}}-\chi_{0, n_{2}}\right\|^{2}+C \int_{0}^{t}\left(\|\widetilde{m}(s)\|^{2}+\|\widetilde{\chi}(s)\|^{2}\right) \mathrm{d} s .
$$

Thus, applying the Gronwall lemma, we obtain that, for all $t \in[0, T]$, the sequence $\left\{\chi_{n}(t)\right\}$ satisfies the Cauchy property in the $H$-norm. Consequently, recalling (3.29) and using once more the Lebesgue theorem, we have

$$
\chi_{n} \rightarrow \chi \quad \text { strongly in } L^{2}(0, T ; H),
$$

whence by the local Lipschitz continuity of $W^{\prime}$ we get $w=W^{\prime}(\chi)$. This concludes the proof of the existence part.

Uniqueness and continuous dependence. Referring to the notation of Theorem 2.2, we set

$$
\widetilde{\vartheta}:=\vartheta_{1}-\vartheta_{2}, \quad \tilde{\chi}:=\chi_{1}-\chi_{2} .
$$

Then, we write both (2.8) and (2.9) for $i=1,2$, take the differences, and test them, respectively, by $\widetilde{\vartheta}$ and $\widetilde{\chi}_{t}$. Noting that a pair of terms cancels out, integrating over $Q_{t}$ for $t \leq T$, adding a relation analogous to (3.39) in order to get the norm of $\widetilde{\chi}$ on the left hand side, recalling (J1), and using the separation property with the local Lipschitz continuity of $W^{\prime}$ (as above) and the Young inequality, it is then easy to get

$$
\begin{aligned}
& \frac{1}{2}\|\widetilde{\vartheta}(t)\|^{2}+\|\nabla \widetilde{\vartheta}\|_{L^{2}(0, t ; H)}^{2}+\frac{\varepsilon}{2}\left\|\widetilde{\chi}_{t}(t)\right\|^{2}+\frac{1}{2}\left\|\widetilde{\chi}_{t}\right\|_{L^{2}(0, t ; H)}^{2}+\|\widetilde{\chi}(t)\|^{2} \leq \frac{1}{2}\left\|\vartheta_{0,1}-\vartheta_{0,2}\right\|^{2} \\
& +\frac{\varepsilon}{2}\left\|\chi_{1,1}-\chi_{1,2}\right\|^{2}+\left\|\chi_{0,1}-\chi_{0,2}\right\|^{2}+C \int_{0}^{t}\|\widetilde{\chi}(s)\|^{2} \mathrm{~d} s
\end{aligned}
$$

where we point out that $C$, depending on $W$ and $\mathcal{J}$, is independent of $\varepsilon$ due to the separation property. Then, the claim follows via the Gronwall lemma.

4. Proof of Theorem 2.6. Let $\varepsilon$ be fixed and let $(\vartheta, \chi)$ be a solution. We shall prove first that (2.20) and (2.21) hold. With assumption (f2) at our disposal, we can come back to (3.27) and note that now the right hand side can be dominated uniformly in time thanks to the Young and Poincaré inequalities. This leads us to the bounds (cf. $(3.28)-(3.30))$

$$
\begin{aligned}
& \|\vartheta\|_{L^{\infty}(0, \infty ; H)}+\|\vartheta\|_{L^{2}(0, \infty ; V)} \leq c \\
& \varepsilon^{1 / 2}\left\|\chi_{t}\right\|_{L^{\infty}(0, \infty ; H)}+\left\|\chi_{t}\right\|_{L^{2}(0, \infty ; H)} \leq c, \\
& \|W(\chi)\|_{L^{\infty}\left(0, \infty ; L^{1}(\Omega)\right)} \leq c .
\end{aligned}
$$

Note indeed that (3.28)-(3.30), which are proved for the approximations, are preserved when we pass to the limit with respect to $n$ by the semicontinuity of the norms with 
respect to the weak and weak star convergences. Proceeding along the lines of the previous section, we can realize that also (3.31) and (3.35) can be made uniform in time, so that

$$
\begin{array}{r}
\|\vartheta\|_{H^{1}(0, \infty ; H)}+\|\vartheta\|_{C^{0}([0, \infty) ; V)}+\|\vartheta\|_{L^{2}\left(0, \infty ; H^{2}(\Omega)\right)} \leq c \\
\|\chi\|_{L^{\infty}(\Omega \times(0, \infty))}+\varepsilon^{1 / 2}\left\|\chi_{t}\right\|_{L^{\infty}(\Omega \times(0, \infty))}+\|W(\chi)\|_{L^{\infty}(\Omega \times(0, \infty))} \leq c
\end{array}
$$

Moreover, differentiating (2.9) in time and testing the result by $\chi_{t t}$, thanks to (4.4) and (4.5) together with (W1) and (J1), it is a standard matter to deduce

$$
\left\|\chi_{t t}\right\|_{L^{2}(0, \infty ; H)} \leq c
$$

Finally, a comparison of terms in the time derivative of (2.9) gives

$$
\varepsilon\left\|\chi_{t t t}\right\|_{L^{2}(0, \infty ; H)} \leq c
$$

From the above estimates, we infer (2.21). As far as $\vartheta$ is concerned, if we multiply equation (2.8) by $\vartheta_{t}$ and integrate over $\Omega$, using the Green's formula, we obtain

$$
\frac{1}{2} \frac{\mathrm{d}}{\mathrm{d} t}\|\nabla \vartheta\|^{2}+\left\|\vartheta_{t}\right\|^{2}=\left(f-\chi_{t}, \vartheta_{t}\right) \leq \frac{1}{2}\left\|\vartheta_{t}\right\|^{2}+\frac{1}{2}\left\|f-\chi_{t}\right\|^{2}
$$

Then, setting $h(t):=\|\nabla \vartheta(t)\|^{2}$, we can deduce (2.20) from (f2), (4.4), (4.5), and [35, Lemma 6.2.1]. Concerning $\chi$, we have, up to a subsequence $\left\{t_{n}\right\}$,

$$
\chi\left(t_{n}\right) \rightarrow \chi_{\infty} \quad \text { weakly star in } L^{\infty}(\Omega),
$$

where $\chi_{\infty} \in L^{\infty}(\Omega)$ is a suitable limit function, depending on $\left\{t_{n}\right\}$. Thus the $\omega$-limit of the solution under consideration is not empty.

Let us now see that $\chi_{\infty}$ is a solution to (1.9). By (4.5), there exists $w \in L^{\infty}(\Omega)$ such that, possibly up to a further extraction, $W^{\prime}\left(\chi\left(t_{n}\right)\right) \rightarrow w$ weakly star in $L^{\infty}(\Omega)$. Moreover, from the linearity and compactness of $\mathcal{J}$ (cf. (J1)-(J2)), we have that $\mathcal{J}\left[\chi\left(t_{n}\right)\right] \rightarrow$ $\mathcal{J}\left[\chi_{\infty}\right]$ strongly in $C(\bar{\Omega})$. Recalling now $(2.20)$ and $(2.21)$, by comparison we readily get $w=\mathcal{J}\left[\chi_{\infty}\right]$. Moreover, the convergence $W^{\prime}\left(\chi\left(t_{n}\right)\right) \rightarrow w$ turns out to be strong in $H$. Thus, to identify $w$ with $W^{\prime}\left(\chi_{\infty}\right)$, we can use $(4.8)$, the monotonicity of $W^{\prime}$, and, e.g., [2, Prop. 1.1, p. 42]. Finally, it is easy to see that, if $W$ is strictly convex (i.e., $\lambda<0$ in (W2)), then $\left(W^{\prime}\right)^{-1}$ is a Lipschitz continuous function. We can thus infer from equation (2.9) that $\chi\left(t_{n}\right) \rightarrow \chi_{\infty}$ strongly in $H$. In other words, the orbit $\bigcup_{t \geq 0}\{\chi(t)\}$ is precompact in $H$.

In order to complete the proof, we must demonstrate that the $\omega$-limit of the solution $(\vartheta, \chi)$ reduces to one point provided that $W$ is strictly convex and $(2.16)$ holds. We can argue as in the proof of [23, Thm. 3.5] (see also [10, Proof of Thm. 2.3]); therefore we just outline the main argument. 
Let $\left(0, \chi_{\infty}\right)$ be in the $\omega$-limit set of our solution $(\vartheta, \chi)$ and suppose $(2.23)$. Then, introduce the unbounded set

$$
\Sigma:=\left\{t \geq 0:\left\|\chi(t)-\chi_{\infty}\right\| \leq \frac{\sigma}{3}\right\}
$$

where $\sigma$ is given by Theorem 2.4. For every $t \in \Sigma$, define

$$
\tau(t):=\sup \left\{t^{\prime} \geq t: \sup _{s \in\left[t, t^{\prime}\right]}\left\|\chi(s)-\chi_{\infty}\right\| \leq \sigma\right\}
$$

and note that $\tau(t)>t$.

On account of (2.20) and (2.21), we can take $t_{0} \in \Sigma$ large enough such that

$$
\|\vartheta(t)\|_{V}+\left\|\chi_{t}(t)\right\| \leq 1, \quad \forall t \geq t_{0}
$$

and consider the sets

$$
\begin{aligned}
J & :=\left[t_{0}, \tau\left(t_{0}\right)\right), \\
J_{1} & :=\left\{t \in J: N(\vartheta, \chi)(t)>\left(\int_{t}^{\infty}\|f(s)\|^{2} \mathrm{~d} s\right)^{1-\rho}\right\}, \\
J_{2} & :=J \backslash J_{1}, \\
J_{3} & :=\{t \geq 0: \beta\|\vartheta(t)\| \leq\|f(t)\|\},
\end{aligned}
$$

where

$$
N(\vartheta, \chi)(t)=\|\vartheta(t)\|+\left\|\chi_{t}(t)\right\|+\left\|-\mathcal{J}[\chi(t)]+W^{\prime}(\chi(t))\right\|, \quad \forall t>0,
$$

and $\beta>0$ is to be fixed later on. For every $t \in J$, we introduce the functional

$$
\begin{aligned}
\mathcal{L}(t) & =\frac{1}{2}\left(\|\vartheta(t)\|^{2}+\varepsilon\left\|\chi_{t}(t)\right\|^{2}\right)+F(\chi(t))-F\left(\chi_{\infty}\right) \\
& +\int_{t}^{\tau\left(t_{0}\right)}(f(s), \vartheta(s)) \Upsilon_{J_{3}}(s) \mathrm{d} s+\alpha \varepsilon\left(\chi_{t}(t),-\mathcal{J}[\chi(t)]+W^{\prime}(\chi(t))\right),
\end{aligned}
$$

$\Upsilon_{J_{3}}$ being the characteristic function of $J_{3}$ and $\alpha>0$ is a small parameter which will be fixed below. Then we observe that

$$
\begin{aligned}
\frac{d}{d t} \mathcal{L} & =-\|\vartheta\|_{V}^{2}-\left\|\chi_{t}\right\|^{2}+(f, \vartheta)\left(1-\Upsilon_{J_{3}}\right) \\
& +\alpha \varepsilon\left(\chi_{t},\left[-\mathcal{J}[\chi]+W^{\prime}(\chi)\right]_{t}\right)-\alpha\left(\chi_{t},-\mathcal{J}[\chi]+W^{\prime}(\chi)\right) \\
& -\alpha\left\|-\mathcal{J}[\chi]+W^{\prime}(\chi)\right\|^{2}+\alpha\left(\vartheta,-\mathcal{J}[\chi]+W^{\prime}(\chi)\right)
\end{aligned}
$$

On account of (W1), (2.2), (J1), and taking advantage of the Poincaré and the Young inequalities, it is not difficult to deduce

$$
\begin{aligned}
& (f, \vartheta)\left(1-\Upsilon_{J_{3}}\right) \leq c \beta\|\vartheta\|_{V}^{2}, \\
& \alpha\left(\chi_{t},\left[-\mathcal{J}[\chi]+W^{\prime}(\chi)\right]_{t}\right) \leq c \alpha\left\|\chi_{t}\right\|^{2} \\
& -\alpha\left(\chi_{t},-\mathcal{J}[\chi]+W^{\prime}(\chi)\right) \leq \frac{\alpha}{4}\left\|-\mathcal{J}[\chi]+W^{\prime}(\chi)\right\|^{2}+c \alpha\left\|\chi_{t}\right\|^{2} \\
& \alpha\left(\vartheta,-\mathcal{J}[\chi]+W^{\prime}(\chi)\right) \leq \frac{\alpha}{4}\left\|-\mathcal{J}[\chi]+W^{\prime}(\chi)\right\|^{2}+c \alpha\|\vartheta\|^{2} .
\end{aligned}
$$


Collecting the above estimates, from (4.9) we infer

$$
\frac{d}{d t} \mathcal{L} \leq-(1-c \alpha-c \beta)\|\vartheta\|_{V}^{2}-(1-c \alpha)\left\|\chi_{t}\right\|^{2}-\frac{\alpha}{2}\left\|-\mathcal{J}[\chi]+W^{\prime}(\chi)\right\|^{2},
$$

which yields, for $\alpha$ and $\beta$ sufficiently small,

$$
\frac{\mathrm{d}}{\mathrm{d} t} \mathcal{L} \leq-c N(\vartheta, \chi)^{2}
$$

Therefore $\mathcal{L}$ is decreasing. This entails that $|\mathcal{L}|^{\rho} \operatorname{sgn} \mathcal{L}$ is decreasing as well, due to the identity

$$
\frac{\mathrm{d}}{\mathrm{d} t}\left(|\mathcal{L}(t)|^{\rho} \operatorname{sgn} \mathcal{L}(t)\right)=\rho|\mathcal{L}(t)|^{\rho-1} \frac{\mathrm{d}}{\mathrm{d} t} \mathcal{L}(t), \quad t \in J .
$$

Using now (2.18), we deduce that, for every $t \in J_{1}$, it holds that

$$
|\mathcal{L}(t)|^{1-\rho} \leq c N(\vartheta, \chi)(t)
$$

Consequently, thanks to (4.11), we infer

$$
\begin{aligned}
\int_{J_{1}} N(\vartheta, \chi)(t) \mathrm{d} t & \leq-c \int_{t_{0}}^{\tau\left(t_{0}\right)} \frac{\mathrm{d}}{\mathrm{d} t}\left(|\mathcal{L}(t)|^{\rho} \operatorname{sgn} \mathcal{L}(t)\right) \\
& \leq c\left(\left|\mathcal{L}\left(t_{0}\right)\right|^{\rho}+\left|\mathcal{L}\left(\tau\left(t_{0}\right)\right)\right|^{\rho}\right)
\end{aligned}
$$

where $\left|\mathcal{L}\left(\tau\left(t_{0}\right)\right)\right|=0$ if $\tau\left(t_{0}\right)=\infty$.

Let now $t \in J_{2}$. Then, on account of (f3), we have

$$
N(\vartheta, \chi)(t) \leq\left(\int_{t}^{\infty}\|f(s)\|^{2} \mathrm{~d} s\right)^{1-\rho} \leq c t^{-(1+\mu)(1-\rho)},
$$

and recalling (2.23), we easily obtain

$$
\int_{J_{2}} N(\vartheta, \chi)(t) \mathrm{d} t \leq c t_{0}^{-\mu+\rho+\rho \mu}
$$

Hence $\left\|\chi_{t}(\cdot)\right\|$ is integrable over $J$. Moreover, we get

$$
\begin{aligned}
0 & \leq \limsup _{t_{0} \in \Sigma, t_{0} \rightarrow \infty} \int_{t_{0}}^{\tau\left(t_{0}\right)}\left\|\chi_{t}(t)\right\| \mathrm{d} t \\
& \leq c \limsup _{t_{0} \in \Sigma, t_{0} \rightarrow \infty}\left(\left|\mathcal{L}\left(t_{0}\right)\right|^{\rho}+\left|\mathcal{L}\left(\tau\left(t_{0}\right)\right)\right|^{\rho}+t_{0}^{-\mu+\rho+\rho \mu}\right)=0 .
\end{aligned}
$$

Here we have used $(2.20),(2.21)$, (f3) and the fact that $F$ takes the same value on any point $\chi_{*}$ such that $\left(0, \chi_{*}\right)$ belongs to the $\omega$-limit set of the solution $(\vartheta, \chi)$.

Observe now that, for every $t \in J$,

$$
\left\|\chi(t)-\chi_{\infty}\right\| \leq \int_{t_{0}}^{t}\left\|\chi_{t}(s)\right\| \mathrm{d} s+\left\|\chi\left(t_{0}\right)-\chi_{\infty}\right\|,
$$

and suppose that $\tau\left(t_{0}\right)<\infty$ for any $t_{0} \in \Sigma$. By definition, we have

$$
\left\|\chi\left(\tau\left(t_{0}\right)\right)-\chi_{\infty}\right\|=\sigma, \quad \forall t_{0} \in \Sigma .
$$

On the other hand, we have proved above that there exists an unbounded sequence $\left\{t_{n}\right\}_{n \in \mathbb{N}} \subset \Sigma$ such that

$$
\lim _{n \rightarrow \infty}\left\|\chi\left(t_{n}\right)-\chi_{\infty}\right\|=0 .
$$


However, due to the precompactness of $\bigcup_{t>0}\{\chi(t)\}$ in $H$, we can find a subsequence $\left\{t_{n_{k}}\right\}_{k \in \mathbb{N}}$ and an element $\tilde{\chi}_{\infty}$ which is a stationary point for $F$ such that $\left\|\tilde{\chi}_{\infty}-\chi_{\infty}\right\|=\sigma$ and

$$
\lim _{k \rightarrow \infty}\left\|\chi\left(\tau\left(t_{n_{k}}\right)\right)-\tilde{\chi}_{\infty}\right\|=0
$$

Thus, from (4.15) and (4.16), we deduce the contradiction

$$
0<\left\|\tilde{\chi}_{\infty}-\chi_{\infty}\right\| \leq \limsup _{k \rightarrow \infty}\left(\int_{t_{n_{k}}}^{\tau\left(t_{n_{k}}\right)}\left\|\chi_{t}(s)\right\| \mathrm{d} s+\left\|\chi\left(t_{n_{k}}\right)-\chi_{\infty}\right\|\right)=0 .
$$

This entails that $\tau\left(t_{0}\right)=\infty$ for some $t_{0}>0$ sufficiently large. Consequently $\left\|\chi_{t}(\cdot)\right\|$ is integrable over $\left(t_{0}, \infty\right)$. Hence, using once again the precompactness of $\bigcup_{t>0}\{\chi(t)\}$ in $H$, we conclude that $\chi(t)$ strongly converges to $\chi_{\infty}$ so that the $\omega$-limit set of $(\vartheta, \chi)$ contains $\left(0, \chi_{\infty}\right)$ only. Of course, when $(2.25)$ holds, we can argue in the same way. To show the convergence estimates (2.24) and (2.26), we can proceed exactly as in [23, Proof of Thm. 3.5].

\section{REFERENCES}

[1] H. Amann, "Linear and Quasilinear Parabolic Problems", Birkhäuser Verlag, Basel-Boston-Berlin, 1995. MR1345385 (96g:34088)

[2] V. Barbu, "Nonlinear Semigroups and Differential Equations in Banach Spaces", Noordhoff, Leyden, 1976. MR0390843 (52:11666)

[3] P.W. Bates, F. Chen, Traveling wave solutions for a nonlocal phase-field system, Interfaces Free Bound., 4 (2002), 227-238. MR1914622 (2003f:35275)

[4] P.W. Bates, F. Chen, Spectral analysis and multidimensional stability of traveling waves for nonlocal Allen-Cahn equation, J. Math. Anal. Appl., 273 (2002), 45-57. MR1933014 (2003h:35104)

[5] P.W. Bates, J. Han, The Neumann boundary problem for a nonlocal Cahn-Hilliard equation, J. Differential Equations, 212 (2005), 235-277. MR2129092 (2005m:35141)

[6] M. Brokate, J. Sprekels, "Hysteresis and Phase Transitions", Springer, New York, 1996. MR1411908 (97g:35127)

[7] G. Caginalp, An analysis of a phase field model of a free boundary, Arch. Rational Mech. Anal., 92 (1986), 205-245. MR816623 (87c:80011)

[8] J.C. Cahn, J.E. Hilliard, Free energy of a nonuniform system. I. Interfacial free energy, J. Chem. Phys., 28 (1958), 258-267.

[9] R. Chill, On the Eojasiewicz-Simon gradient inequality, J. Funct. Anal., 201 (2003), 572-601. MR1986700 (2005c:26019)

[10] R. Chill, M.A. Jendoubi, Convergence to steady states in asymptotically autonomous semilinear evolution equations, Nonlinear Anal., 53 (2003), 1017-1039. MR1978032 (2004d:34103)

[11] E. Feireisl, F. Simondon, Convergence for semilinear degenerate parabolic equations in several space dimensions, J. Dynam. Differential Equations, 12 (2000), 647-673. MR1800136 (2002g:35116)

[12] E. Feireisl, F. Issard-Roch, H. Petzeltová, A non-smooth version of the Eojasiewicz-Simon theorem with applications to non-local phase-field systems, J. Differential Equations, 199 (2004), 1-21. MR2041509 (2005c:35284)

[13] H. Gajewski, On a nonlocal model of non-isothermal phase separation, Adv. Math. Sci. Appl., 12 (2002), 569-586. MR1943981 (2003k:35103)

[14] H. Gajewski, K. Zacharias, On a nonlocal phase separation model, J. Math. Anal. Appl., 286 (2003), 11-31. MR2009615 (2004i:35163)

[15] P. Galenko, D. Jou, Diffuse-interface model for rapid phase transformations in nonequilibrium systems, Phys. Rev. E, 71 (2005), 046125(13).

[16] G. Giacomin, J.L. Lebowitz, Phase segregation dynamics in particle systems with long range interactions. I. Macroscopic limits, J. Statist. Phys., 87 (1997), 37-61. MR1453735 (98m:82053)

[17] G. Giacomin, J.L. Lebowitz, Phase segregation dynamics in particle systems with long range interactions. II. Interface motion, SIAM J. Appl. Math., 58 (1998), 1707-1729 (electronic). MR1638739 (99m:35249) 
[18] C. Giorgi, M. Grasselli and V. Pata, Uniform attractors for a phase field model with memory and quadratic nonlinearity, Indiana Univ. Math. J., 48 (1999), 1395-1445. MR1757078 (2001h:37160)

[19] M. Grasselli, A. Miranville, V. Pata, S. Zelik, Well-posedness and long time behavior of a parabolichyperbolic phase-field system with singular potentials, Math. Nachr., to appear.

[20] M. Grasselli, V. Pata, Existence of a universal attractor for a parabolic-hyperbolic phase-field system, Adv. Math. Sci. Appl., 13 (2003), 443-459. MR2029927 (2004k:37172)

[21] M. Grasselli, V. Pata, Asymptotic behavior of a parabolic-hyperbolic system, Commun. Pure Appl. Anal., 3 (2004), 849-881. MR2106302 (2005h:35150)

[22] M. Grasselli, H. Petzeltová, G. Schimperna, Long time behavior of solutions to the Caginalp system with singular potential, Z. Anal. Anwendungen, 25 (2006), 51-72. MR2216881 (2007b:35159)

[23] M. Grasselli, H. Petzeltová, G. Schimperna, Convergence to stationary solutions for a parabolichyperbolic phase-field system, Commun. Pure Appl. Anal., 5 (2006), 827-838. MR2246010

[24] S.-Z. Huang, P. Takáč, Convergence in gradient-like systems which are asymptotically autonomous and analytic, Nonlinear Anal., 46 (2001) 675-698. MR1857152 (2002f:35125)

[25] M.A. Jendoubi, A simple unified approach to some convergence theorems of L. Simon, J. Funct. Anal., 153 (1998), 187-202. MR1609269 (99c:35101)

[26] P. Krejčí, J. Sprekels, Nonlocal phase-field models for non-isothermal phase transitions and hysteresis, Adv. Math. Sci. Appl., 14 (2004), 593-612. MR2111831 (2006d:74063)

[27] P. Krejčí, J. Sprekels, Long time behaviour of a singular phase transition model, Discrete Contin. Dyn. Syst., 15 (2006), 1119-1135. MR2224500

[28] P. Krejčí, E. Rocca, J. Sprekels, Nonlocal temperature-dependent phase-field models for nonisothermal phase transitions, WIAS preprint n. 1006, Berlin (2005).

[29] S. Łojasiewicz, Une propriété topologiqque des sous-ensembles analytiques réels, in Colloques internationaux du C.N.R.S. 117: Les équations aux dérivées partielles (Paris, 1962), 87-89. Editions du C.N.R.S., Paris, 1963. MR0160856 (28:4066)

[30] S. Łojasiewicz, "Ensembles Semi-analytiques", notes, I.H.E.S., Bures-sur-Yvette, 1965.

[31] L. Simon, Asymptotics for a class of non-linear evolution equations, with applications to geometric problems, Ann. of Math. (2), 118 (1983), 525-571. MR727703 (85b:58121)

[32] J. Sprekels, S. Zheng, Global existence and asymptotic behaviour for a nonlocal phase-field model for non-isothermal phase transitions, J. Math. Anal. Appl., 279 (2003), 97-110. MR1970493 (2004c:45015)

[33] X. Wang, Metastability and stability of patterns in a convolution model for phase transitions, J. Differential Equations, 183 (2002), 434-461. MR1919786 (2003f:35157)

[34] H. Wu, M. Grasselli, S. Zheng, Convergence to equilibrium for a parabolic-hyperbolic phase-field system with Neumann boundary conditions, Math. Models Methods Appl. Sci., 17 (2007), 125-153. MR2290411

[35] S. Zheng, "Nonlinear Evolution Equations", Chapman \& Hall/CRC, Boca Raton, Florida, 2004. MR2088362 (2006a:35001) 